\title{
Morbidity and mortality in type B Niemann-Pick disease
}

\author{
Margaret M. McGovern, MD, PhD'1, Natalie Lippa, MS, CGC², Emilia Bagiella, PhD², \\ Edward H. Schuchman, PhD², Robert J. Desnick, PhD, MD² and Melissa P. Wasserstein, MD²
}

Purpose: The purpose of this study was to perform a systematic evaluation of morbidity and mortality in type B Niemann-Pick disease.

Methods: A total of 103 patients with Niemann-Pick disease (49 males, 54 females, age range: 1-72 years) participated in natural history studies through Mount Sinai's International Center for Types A and B Niemann-Pick Disease between 1992 and 2012.

Results: Serious morbidities included significant neurological, hepatic, and cardiac disease. Thirteen patients had some degree of neurological impairment. Nine patients had cirrhosis or liver failure requiring transplantation. Coronary artery and valvular heart disease were present in nine patients. Of note, only four patients were oxygen dependent, although progressive pulmonary disease is a well-described feature of Niemann-Pick disease. During the

Niemann-Pick disease (NPD) is a rare lysosomal storage disease caused by deficient activity of acid sphingomyelinase (ASM; sphingomyelin phosphodiesterase; EC 3.1.4.12) and the accumulation of sphingomyelin within cells of the monocytemacrophage system. ${ }^{1}$ Types A and B NPD are inherited as autosomal recessive traits, and result from allelic mutations in the ASM gene (SMPD1). Type A disease, which has an Ashkenazi Jewish predilection, is a fatal neurodegenerative disorder of infancy. In contrast, type B NPD (NP-B), which is panethnic, is characterized by phenotypic heterogeneity and the absence of central nervous system involvement in most patients. Type $\mathrm{C}$ disease is biochemically, genetically, and clinically distinct from type A disease and NP-B and is caused by mutations in the NPC1 or NPC2 genes, which results in a disruption of intracellular cholesterol transport.

Common clinical manifestations of NP-B are hepatosplenomegaly, hyperlipidemia, and infiltrative pulmonary disease. Other features that have been reported include liver dysfunction, cardiac disease, retinal stigmata, growth retardation, and skeletal manifestations including osteoporosis and osteopenia. ${ }^{2-7}$ In addition, there is a subset of variant patients who survive early childhood but have progressive neurologic findings including ataxia, variable degrees of developmental delay, and peripheral neuropathy. ${ }^{89}$ Accompanying this marked phenotypic variability among patients with NP-B is a broad range of disease severity. For example, age at clinical presentation can range from early childhood to the fourth or fifth decades of life. follow-up period, 18 deaths occurred. The median age of death was 15.5 years (range 1-72). Causes of death included pneumonia, liver failure, and hemorrhage. The majority of deaths (12 of 18) occurred in patients $<21$ years, yielding a mortality rate of $19 \%$ in the pediatric population.

Conclusion: This study demonstrates that Niemann-Pick disease is a life-threatening disorder with significant morbidity and mortality, especially in the pediatric population. The information collected in this series highlights the need for safe, effective therapy for Niemann-Pick disease.

Genet Med 2013:15(8):618-623

Key Words: acid sphingomyelinase deficiency; morbidity; mortality; Niemann-Pick disease

Some patients have massive organomegaly, whereas others have only minimal splenic enlargement. ${ }^{10}$

Although there have been single case reports of deaths in patients with NP-B, to date there has been no systematic evaluation of the causes of mortality. In this article, we summarize the major morbidities in a series of 103 patients with NP-B who were evaluated at our center and describe the age and cause of death in 18 patients who died during the course of the study.

\section{Study subjects}

\section{MATERIALS AND METHODS}

A total of 103 patients with NP-B (49 males, 54 females, age range: $1-72$ years) participated in natural history studies as part of Mount Sinai's International Center for Types A and B Niemann-Pick Disease. These studies were carried out at the Mount Sinai General Clinical Research Center between 1992 and 2012. The studies were approved by the Mount Sinai School of Medicine Institutional Review Board. All participants gave voluntary, written informed consent and minor assent as appropriate to participate and to allow the study team to maintain contact with them. The diagnosis of NP-B was confirmed in each patient by the demonstration of reduced ASM activity in isolated leukocytes and/or cultured skin fibroblasts and by the identification of two diseasecausing ASM mutations as previously described. ${ }^{10}$ At entry, 61 patients were in the pediatric age group $(<21$ years of age) and 42 were adults. The upper cutoff for the pediatric age

${ }^{1}$ Department of Pediatrics, Stony Brook School of Medicine, Stony Brook, New York, USA; ${ }^{2}$ Department of Genetics and Genomic Sciences, Mount Sinai School of Medicine,

New York, New York, USA. Correspondence: Melissa P. Wasserstein (Melissa.wasserstein@mssm.edu) 
group was 21 years of age because many patients with NP-B have delayed puberty. ${ }^{6}$ The majority of patients were of mixed European/Caucasian descent; several were Ashkenazi Jewish; and others were of Middle Eastern or Hispanic/Caucasian descent. To calculate age at last follow-up, we used age at last visit for living patients or age at death for deceased patients. Table 1 provides an overview of the characteristics of the living and deceased patients.

\section{Clinical studies}

Detailed medical histories were obtained for all patients on entry into the study including age at diagnosis, information about previous medical and surgical histories, concurrent illness, and splenectomy status. Interim medical history also was obtained at the time of each subsequent visit. In addition, patients frequently contacted the investigators between visits when their clinical status changed. All patients underwent a complete physical examination at the time of each visit as well as extensive clinical evaluation, as has been previously reported..$^{10,11}$ Results of quantitative magnetic resonance imaging or computed tomography scans to measure liver and spleen volumes were available for 48 patients.

\section{Causes of death}

Causes of death were learned about from family members or the local treating physician. In some cases, families shared postmortem reports with us. However, some of the deceased patients did not have autopsies, so family member-reported data were used for "cause of death."

\section{Statistical analysis}

All data are displayed as means and SDs for continuous variables and frequency and percentages for categorical variables. Kaplan-Meier curves were constructed to estimate the cumulative survival probability for the whole sample and the pediatric patients.

\section{Table 1 Patient demographics}

Deceased $(n=18) \quad$ Alive $(n=85)$

\begin{tabular}{lcc}
\hline Age at diagnosis & & \\
Median (range) & $2(0.5-53)$ & $4(1-53)$ \\
Mean (SD) & $7.5(15)$ & $8.7(11.4)$ \\
Age at death or last follow-up & & \\
Range & $2-72$ & $2-57$ \\
Mean (SD) & $25(24.4)$ & $22(14.8)$ \\
Median & 17 & 19 \\
Gender & 9 & 40 \\
Male & 9 & 45 \\
Female & & \\
Spleen status & 4 & 3 \\
Total splenectomy & 1 & 81 \\
Partial splenectomy & 13 & \\
Intact &
\end{tabular}

\section{Morbidity}

\section{RESULTS}

All affected patients in the cohort displayed some combination of the common clinical manifestations of NP-B, including splenomegaly, thrombocytopenia, interstitial lung disease, atherogenic lipid profile, osteoporosis or osteopenia, and short stature. Eight had a constellation of neurologic findings including gross motor delay, ataxia, and learning disability that placed them in the variant NP-B category. In addition to these typical disease manifestations, other significant clinical events occurred in many patients and are summarized in Table 2.

Six patients had fulminant liver failure and in addition, three others had evidence of cirrhosis on liver biopsy. Other causes of hepatic injury, such as chronic hepatitis, were excluded. Of those with liver failure, two received successful orthotopic liver transplants at 12 and 25 years of age and remain alive and the third patient is being evaluated for transplant. The remaining three, none of whom received a transplant, died from liver failure, including a 16-year-old girl who also had acute promyelocytic leukemia. Five patients had moderate to severe valvular heart disease. One of these patients required an aortic valvuloplasty and died in the postoperative period of uncontrollable bleeding; another received an aortic valve replacement at the age of 26 years but ultimately died of heart failure in her 60s; and the third had both mitral and aortic valve replacements in adulthood and died decades later from pneumonia. Four patients (age range 30s-50s) had significant coronary artery disease, one of whom underwent bypass surgery in his early 40 s.

Seven patients had clinically significant bleeding: a teenager had recurrent episodes of epistaxis severe enough to necessitate several hospitalizations and multiple blood transfusions; two patients had epidural bleeds after childhood trauma; two other patients had subdural bleeds, one of which was fatal; and one patient died from postoperative bleeding after valvular heart surgery. One patient was also diagnosed with von Willebrand disease. All four patients who had oxygen-dependent pulmonary disease died during the course of the study, two from pneumonia. One patient was diagnosed with systemic lupus erythematosis at about 21 years of age and developed petit mal seizures several years later. Another had recurrent bouts of idiopathic thrombocytopenic purpura. Three patients had cholelithiasis requiring removal of the gallbladder. Pathologic skeletal disease was present in three patients. Of the three patients, one suffered multiple pathologic compression fractures of the spine starting in her 40s, another had recurrent lower extremity fractures in the first 2 years of life, and the third had two pathologic fractures in adulthood. Six patients had significant psychiatric disease, including two with major depression requiring inpatient hospitalization. Two patients were diagnosed with cancer, a 16-year-old girl with acute promyelocytic leukemia and a woman who developed liver cancer at the age of 64 .

\section{Mortality}

As summarized in Table 1, 18 patients died during the study period. Age of death ranged from 2 to 72 years of age; the 
Table 2 Major morbidities in living and deceased patients

\begin{tabular}{|c|c|c|}
\hline Type of morbidity & $\begin{array}{l}\text { Living } \\
(n=85)\end{array}$ & $\begin{array}{c}\text { Deceased } \\
(n=18)\end{array}$ \\
\hline \multicolumn{3}{|l|}{ Neurological disease (13) } \\
\hline Variant Niemann-Pick B & 1 & 7 \\
\hline Ataxia, isolated & 1 & \\
\hline Petit mal seizures & 1 & \\
\hline Learning disabilities & 2 & \\
\hline Sensory dyspraxia & & 1 \\
\hline \multicolumn{3}{|l|}{ Liver disease (9) } \\
\hline Liver failure $(n=6)$ & 3 & 3 \\
\hline Cirrhosis $(n=3)$ & 3 & \\
\hline \multicolumn{3}{|l|}{ Cardiac disease (9) } \\
\hline Valvular heart disease $(n=5)$ & 2 & 3 \\
\hline Coronary artery disease $(n=4)$ & 4 & \\
\hline \multicolumn{3}{|l|}{ Bleeding disorders (7) } \\
\hline Postoperative hemorrhage $(n=1)$ & & 1 \\
\hline Epidural hematoma $(n=2)$ & 1 & \\
\hline Subdural hematoma $(n=2)$ & 1 & 1 \\
\hline Severe epistaxis $(n=1)$ & 1 & \\
\hline Von Willebrand disease $(n=1)$ & 1 & \\
\hline Oxygen-dependent pulmonary disease (4) & & 4 \\
\hline \multicolumn{3}{|l|}{ Autoimmune disease (2) } \\
\hline Systemic lupus erythematosus $(n=1)$ & 1 & \\
\hline Idiopathic thrombocytopenic purpura $(n=1)$ & 1 & \\
\hline Cholelithiasis (3) & 2 & 1 \\
\hline Pathologic skeletal fractures $(n=3)$ & 2 & 1 \\
\hline \multicolumn{3}{|l|}{ Psychiatric disease (6) } \\
\hline Depression $(n=4)$ & 3 & 1 \\
\hline Bipolar disease $(n=1)$ & 1 & \\
\hline Attention deficit hyperactivity disorder $(n=1)$ & & 1 \\
\hline \multicolumn{3}{|l|}{ Malignancy (2) } \\
\hline Acute promyelocytic leukemia $(n=1)$ & & 1 \\
\hline Liver cancer $(n=1)$ & & 1 \\
\hline
\end{tabular}

mean age of death was 25 years and the median age of death was 17 years. Of the 18 patients who died, $12(67 \%)$ were $\leq 21$ years of age, which represented $19 \%$ of the pediatric cohort. Six of the deaths occurred in patients older than 21, representing $14 \%$ mortality in the adult population. Altogether, 9 of 54 females (16.7\%) and 9 of 49 males (18\%) died. The median age at diagnosis in the live population was 4 years versus 2 years in the deceased population. Figure 1a shows the percentage of patients surviving over time for the entire NP-B cohort. The majority of deaths occurred in patients younger than 21 years of age. Figure $1 \mathrm{~b}$ shows the survival distribution function for the pediatric population.

\section{Causes of death}

The age at death, cause of death, spleen status, and major morbidities for the 18 patients who died are summarized in Table 3 . The most common cause of death was pneumonia/respiratory
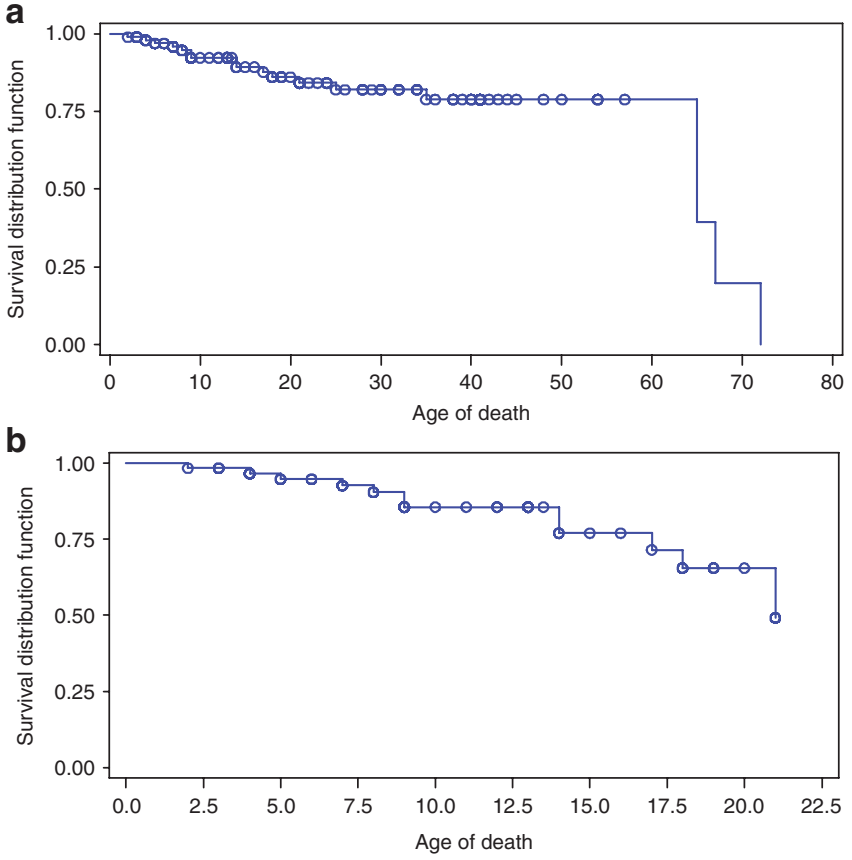

Figure 1 Survival distribution. (a) Survival distribution in all patients. (b) Survival distribution in pediatric patients.

failure $(n=5)$. Three patients died from liver failure and three died from complications following bone marrow transplant. The following were the causes of death in one patient each: postoperative bleeding, splenic vein tear, liver cancer, low-output heart failure, multiorgan failure, and subdural bleed. The cause of death was undetermined in one patient.

\section{Associations with spleen status}

Splenectomy status was recorded in all patients, and quantitative spleen volume was obtained in 48 of them. In our study, seven patients with NP-B had undergone total splenectomy. Four of the seven splenectomized patients died (57\%), as compared with 14 of the 95 non-splenectomized patients (14\%; difference not statistically significant). The mean spleen volume was 10.7 (range 3.6-26.5) multiples of normal in non-deceased patients versus 13.3 multiples of normal (range 8.5-18.2) in the two deceased patients who had spleen volume measurements (difference not statistically significant). Liver function was not significantly different in splenectomized versus non-splenectomized patients.

\section{Associations with SMPD1 genotype}

Genotype was recorded for 16 of the 18 deceased patients and 82 of 85 nondeceased patients. Because most patients were compound heterozygotes, there were 70 different alleles, which made genotype/phenotype correlations difficult. The most common allele, $\Delta \mathrm{R} 608$, was present in a homozygous or heterozygous state in 30 live patients and in a heterozygous state in one deceased patient, who died from NPD-associated liver failure at 25 years of age. This is in concordance with previous reports that $\Delta \mathrm{R} 608$ is associated with non-neuronopathic, milder disease. ${ }^{6}$ 
Table 3 Cause of death, splenectomy status, and major morbidities in deceased patients

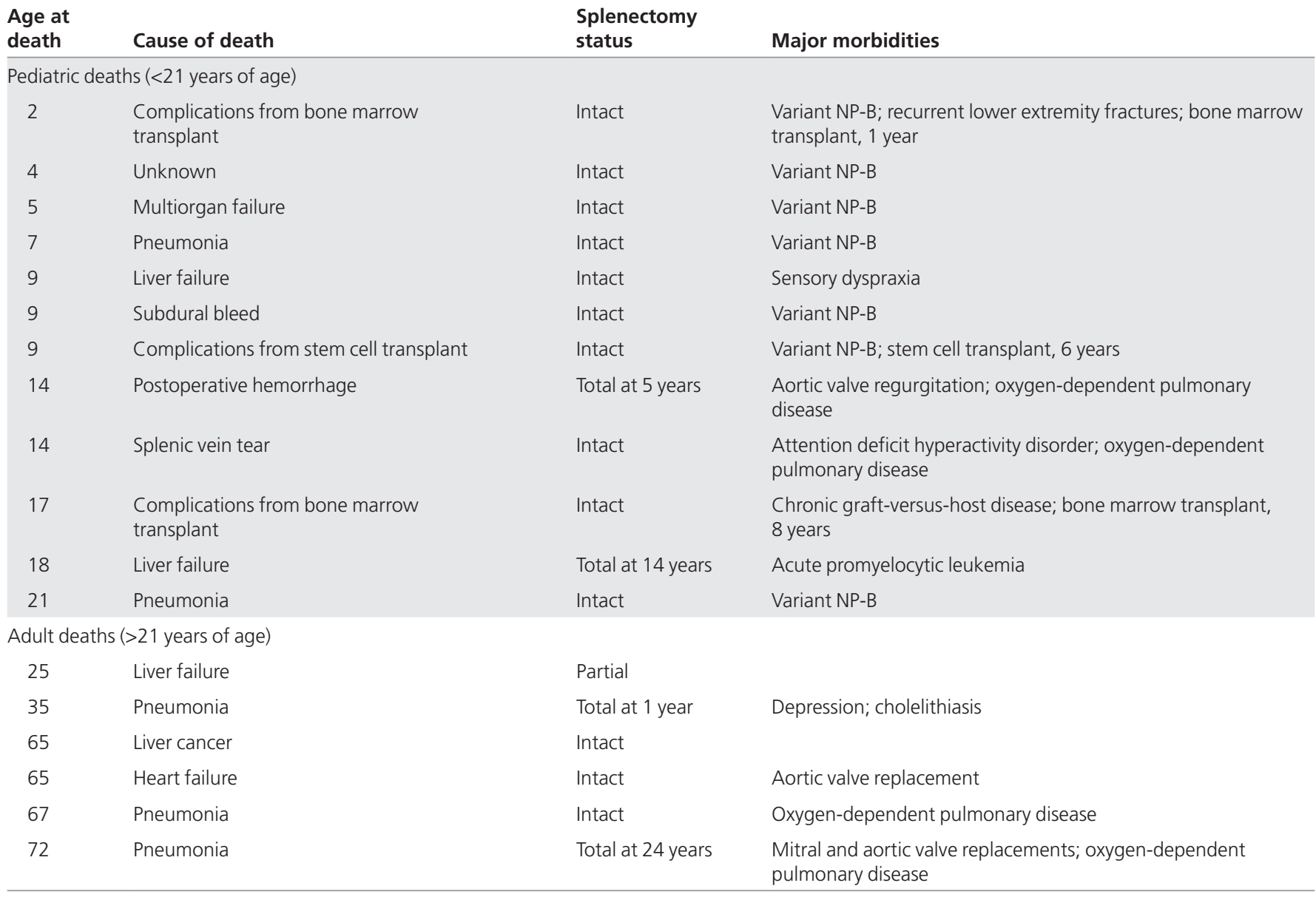

NP-B, type B Niemann-Pick disease.

The Q292K mutation, which has been associated with variant type B NPD, ${ }^{9}$ was present in homozygosity in one deceased patient and in the heterozygous state in one deceased and one alive patient. Both of these deceased patients had variant NP-B, but the patient who is alive has no neurological involvement.

\section{DISCUSSION}

In this article, we provide the first report of the serious and lifethreatening morbidities and causes of death in a large cohort of 103 patients with NP-B who have been followed at the Mount Sinai International Center for Types A and B Niemann-Pick Disease. Previously, reports of morbidity and mortality in this population have been limited to single case reports. Therefore, this study provides the first detailed description of the major morbidities and causes of death in NP-B.

Serious morbidities included clinically significant hepatic, cardiac, and pulmonary disease, each of which also contributed to some patient deaths. With regard to liver involvement, although most affected patients with NP-B manifest stable elevations of hepatic transaminases and bilirubin, ${ }^{11}$ in the series reported here, nine patients (8.7\%) had clinically significant liver disease. In addition to two pediatric patients who died from liver failure, one adult also succumbed to end-stage liver disease, two others had acute liver failure but underwent successful liver transplantation, and a third living patient is currently undergoing evaluation for transplantation; three others had histologic evidence of cirrhosis. Of note, four of the six patients with liver failure presented with this finding in childhood and the remaining two presented in early adulthood, suggesting that there may be a subset of patients who are at increased risk for early, fulminant liver failure. Indeed, severe liver disease in NP-B has been previously reported in children with fatal hepatic failure ${ }^{12}$ and in adults with cirrhosis and portal hypertension. ${ }^{13}$ More recently, 17 patients with NP-B underwent liver biopsies during screening for a phase 1 single ascending dose clinical trial of rhASM, and 15 (88\%) were found to have hepatic fibrosis ranging from grade 1 to grade 4 (frank cirrhosis). ${ }^{14}$ On the basis of these observations it is possible that pathologic intralysosomal storage of sphingomyelin may provoke a fibrotic reaction in the surrounding tissues, leading to progressive liver dysfunction, possibly compounded by inflammation. Given these findings and the observations noted in the patient population reported here, it is reasonable to conclude that liver disease is a significant cause of morbidity and mortality in NP-B.

Cardiac disease included valvular heart disease as well as coronary artery disease. The latter is presumably related to the 
atherogenic lipid profile found in most affected patients with $\mathrm{NP}-\mathrm{B},{ }_{15}^{15}$ whereas the precise mechanism for valvular heart disease is not known. In one patient who underwent valve replacement, progressive heart failure was present that ultimately led to her death, and a pediatric patient with valvular heart disease died of postoperative bleeding.

Psychiatric disease was present in six patients, with two requiring inpatient hospitalization for major depression. Although the overall prevalence $(6 \%)$ is similar to that of the general population, further studies are necessary to assess the severity and potential relationship to NP-B.

Of interest, only four of the patients studied had oxygendependent pulmonary disease, despite the fact that progressive pulmonary disease is a well-described feature of NP-B. Pulmonary function testing typically shows a restrictive pattern of lung involvement with abnormal diffusing capacity, compatible with interstitial lung disease. ${ }^{8}$ Chest radiographs in affected patients reveal interstitial infiltrates with reticulonodular changes and areas of ground-glass density that may be out of proportion to clinical findings. ${ }^{16}$ Severely affected patients may exhibit cough, shortness of breath, and recurrent respiratory infections, ${ }^{17}$ and some patients develop chronic oxygen dependence with progressive pulmonary failure. ${ }^{18}$ The pathophysiology of the pulmonary disease is presumably related to the accumulation of sphingomyelin in the alveolar macrophages, although studies in the ASM knockout mouse also have shown that inflammation, abnormal surfactant catabolism, and abnormal surfactant composition all contribute to lung abnormalities. ${ }^{19,20}$ Similarly, lung biopsies in adults with NP-B have shown endogenous lipid pneumonia, interstitial fibrosis, and accumulation of foamy macrophages. ${ }^{21}$ Therefore, the lung is a target organ of the disease and contributes to the morbidity and mortality in patients with NP-B. Notably, two of the four oxygen-dependent patients in this series also required heart valve replacements, and all four are deceased.

Of the 18 deaths, the majority $(n=12)$ occurred in patients $<21$ years of age, yielding a mortality rate of $19 \%$ in the pediatric population. The survival curve suggests that there are two cohorts of patients: those with more severe disease who have a higher mortality rate during childhood and those with milder disease who are more likely to survive into adulthood. This high mortality rate provides evidence that NP-B is a serious, potentially life-threatening pediatric disorder. Five of the pediatric patients succumbed to complications directly attributable to their disease: fulminant liver failure $(n=2)$ and pneumonia $(n$ $=3$ ). However, three of the 12 pediatric deaths resulted from complications of bone marrow or stem cell transplants, suggesting that the risk-benefit ratio of these procedures must be carefully considered in this patient population. Of note, seven of the 12 pediatric deaths occurred in children with variant NP-B, suggesting that neurological involvement in pediatric patients with NPD B may be associated with early mortality, possibly because of increased overall disease severity in these children. Also notable was the finding that three deaths resulted from bleeding, one from uncontrollable postoperative hemorrhage, another from subdural hematoma suffered after horseplay with a sibling, and the third from a catastrophic abdominal bleed from a torn splenic vein.

We also investigated whether patients with NP-B who had undergone splenectomy had higher mortality than non-splenectomized patients. Splenomegaly is a typical feature in NP-B, ranging from minimal enlargement to $>30$ multiples of normal. ${ }^{10,11}$ Biopsies of the spleen in patients with NP-B demonstrate infiltration with foamy cells, representing sphingomyelin storage within the splenic macrophages. The degree of enlargement may thus represent the body's sphingomyelin burden and overall disease severity, and we have previously demonstrated correlations between the degree of splenomegaly and pancytopenia, pediatric growth restriction, skeletal involvement, and pulmonary compromise. ${ }^{10}$ Of note, $57 \%$ of the splenectomized patients in our cohort died, versus 15\% of those with intact spleens, suggesting an association, although the sample size was not large enough to achieve statistical significance. The deaths in the splenectomized patients were due to variable causes: two adult patients died of pneumonia, one child died from postoperative bleeding during aortic valve replacement, and a teenager died from liver failure and leukemia. Thus, it is possible that the observed higher incidence of death in the splenectomized population is due to chance or reflects the presence of more severe systemic disease among patients referred for splenectomy. However, it is also possible that splenectomy is an independent risk factor for mortality in patients with NP-B. In our cohort, all four splenectomized patients who died had their spleens removed in childhood on the advice of their local physicians because of the degree of splenomegaly rather than any definitive medical indication such as severe thrombocytopenia. As has been observed in splenectomized patients with type 1 Gaucher disease, who have been shown to have lower bone densities, a greater risk of life-threatening pulmonary hypertension, and a lower life expectancy as compared with nonsplenectomized Gaucher patients, it is possible that removal of the spleen eliminates an important reservoir for substrate accumulation that leads to acceleration of disease in other organs such as the lung. ${ }^{22-24}$ Given the limited information currently available on NP-B, it is difficult to determine whether splenectomy directly impacts the progression of disease.

In conclusion, this study demonstrates that NP-B is a lifethreatening disorder associated with significant morbidity and mortality, especially in the pediatric population. The information collected in this series highlights the need for safe, effective therapy for NP-B and suggests that the goals of treatment should be to reduce splenomegaly, improve liver function, and improve respiratory status, with the ultimate goal of reducing serious morbidity and mortality.

\section{ACKNOWLEDGMENT}

M.P.W. is the recipient of a Mentored Patient-Oriented Research Career Development Award (K23 RR16052-01) from the National Institutes of Health (NIH). M.M.M. is the recipient of Mid Career Patient-Oriented Research Career Development Award K24 
RR021991 from the NIH. NPD research in Dr. Schuchman's laboratory is supported by NIH MERIT Award R37 HD28607 and a grant from the Genzyme Corporation. R.J.D. receives research support and financial compensation from Genzyme and Amicus Therapeutics as a consultant. Several patients participated in clinical research studies supported by Genzyme Corporation (Cambridge, MA). These studies were also supported by grant 5 MO1 RR00071 for the Mount Sinai General Clinical Research Center from the National Center for Research Resources, NIH.

\section{DISCLOSURE}

E.H.S. and R.J.D. are inventors on patents owned by the Mount Sinai School of Medicine and licensed to Genzyme for the development of enzyme replacement therapy for the treatment of Niemann-Pick disease. They and the Mount Sinai School of Medicine could receive financial benefit from this license. They are also consultants and have received research grants from Genzyme. The other authors declare no conflict of interest.

\section{REFERENCES}

1. Schuchman E, Desnick RJ. Niemann-Pick disease types a and b: acid sphingomyelinase deficiency. In: Scriver CR, Beaudet AL, Sly WS, Valle D (eds). The Metabolic and Molecular Bases of Inherited Disease. McGraw-Hill: New York, 2001.

2. Tassoni JP Jr, Fawaz KA, Johnston DE. Cirrhosis and portal hypertension in a patient with adult Niemann-Pick disease. Gastroenterology 1991;100:567569.

3. Lever AM, Ryder JB. Cor pulmonale in an adult secondary to Niemann-Pick disease. Thorax 1983:38:873-874.

4. McGovern MM, Wasserstein MP, Aron A, Desnick RJ, Schuchman EH, Brodie SE. Ocular manifestations of Niemann-Pick disease type B. Ophthalmology 2004;111:1424-1427.

5. Lipson MH, O'Donnell J, Callahan JW, Wenger DA, Packman S. Ocular involvement in Niemann-Pick disease type B. J Pediatr 1986;108:582-584.

6. Wasserstein MP, Larkin AE, Glass RB, Schuchman EH, Desnick RJ, McGovern MM. Growth restriction in children with type B Niemann-Pick disease. J Pediatr 2003;142:424-428.

7. Wasserstein M, Godbold J, McGovern MM. Skeletal manifestations in pediatric and adult patients with Niemann Pick disease type B. J Inherit Metab Dis 2013;36:123-127.
8. Wasserstein MP, Aron A, Brodie SE, Simonaro C, Desnick RJ, McGovern MM. Acid sphingomyelinase deficiency: prevalence and characterization of an intermediate phenotype of Niemann-Pick disease. J Pediatr 2006;149:554-559.

9. Pavlu-Pereira H, Asfaw B, Poupctová H, et al. Acid sphingomyelinase deficiency. Phenotype variability with prevalence of intermediate phenotype in a series of twenty-five Czech and Slovak patients. A multi-approach study. J Inherit Metab Dis 2005;28:203-227.

10. McGovern MM, Wasserstein MP, Giugliani R, et al. A prospective, crosssectional survey study of the natural history of Niemann-Pick disease type B. Pediatrics 2008;122:e341-e349.

11. Wasserstein MP, Desnick RJ, Schuchman EH, et al. The natural history of type B Niemann-Pick disease: results from a 10-year longitudinal study. Pediatrics 2004;114:e672-e677.

12. Labrune $P$, Bedossa $P$, Huguet $P$, Roset $F$, Vanier MT, Odievre M. Fatal liver failure in two children with Niemann-Pick disease type B. J Pediatr Gastroenterol Nutr 1991;13:104-109.

13. Putterman C, Zelingher J, Shouval D. Liver failure and the sea-blue histiocyte/ adult Niemann-Pick disease. Case report and review of the literature. J Clin Gastroenterol 1992;15:146-149.

14. Thurberg BL, Wasserstein MP, Schiano T, et al. Liver and skin histopathology in adults with acid sphingomyelinase deficiency (Niemann-Pick disease type B). Am J Surg Path 2012;36:1234-1246.

15. McGovern MM, Pohl-Worgall T, Deckelbaum RJ, et al. Lipid abnormalities in children with types A and B Niemann Pick disease. J Pediatr 2004;145:77-81.

16. Niggemann B, Rebien W, Rahn W, Wahn U. Asymptomatic pulmonary involvement in 2 children with Niemann-Pick disease type B. Respiration 1994:61:55-57.

17. Minai OA, Sullivan EJ, Stoller JK. Pulmonary involvement in Niemann-Pick disease: case report and literature review. Respir Med 2000;94:1241-1251.

18. Guillemot N, Troadec C, de Villemeur TB, Clément A, Fauroux B. Lung disease in Niemann-Pick disease. Pediatr Pulmonol 2007:42:1207-1214.

19. Dhami R, HeX, Gordon RE, Schuchman EH. Analysis of the lung pathology and alveolar macrophage function in the acid sphingomyelinase-deficient mouse model of Niemann-Pick disease. Lab Invest 2001;81:987-999.

20. Ikegami M, Dhami R, Schuchman EH. Alveolar lipoproteinosis in an acid sphingomyelinase-deficient mouse model of Niemann-Pick disease. Am J Physiol Lung Cell Mol Physiol 2003;284:L518-L525.

21. Nicholson AG, Florio R, Hansell DM, et al. Pulmonary involvement by NiemannPick disease. A report of six cases. Histopathology 2006;48:596-603.

22. Pastores GM, Wallenstein S, Desnick RJ, Luckey MM. Bone density in Type 1 Gaucher disease. J Bone Miner Res 1996:11:1801-1807.

23. Weinreb NJ, Deegan P, Kacena KA, et al. Life expectancy in Gaucher disease type 1. Am J Hematol 2008;83:896-900.

24. Mistry PK, Sirrs S, Chan A, et al. Pulmonary hypertension in type 1 Gaucher's disease: genetic and epigenetic determinants of phenotype and response to therapy. Mol Genet Metab 2002;77:91-98. 